\title{
Magnetic properties of single-domain particles and the rock remanence
}

\author{
L. L. Afremov, and V. I. Belokon \\ Far East State University, Vladivostok, Russia \\ Received 15 December 2004; revised 10 March 2005; accepted 1 May 2005; published 24 September 2005.
}

[1] Magnetic states of systems of interacting heterogeneous particles modeling a ferromagnetic rock are theoretically studied. Main attention is given to such important characteristics of the systems as the spontaneous magnetization, the critical field of an irreversible change in the grain magnetic moment, and the distribution of interaction fields. It is shown that particles with strongly imperfect lattices are characterized by a minimum concentration of "magnetic" ions $n=n_{0}$ (a percolation limit) enabling the formation of spontaneous magnetization. This magnetization and the Curie point depend on $n$ and tend toward values of a "pure" ferromagnet. Active diffusion of magnetic ions can locally increase the concentration to values grater than $n>n_{0}$, because the concentration phase transition is absent if diffusion is taken into account. Analysis of magnetic states of a single-domain grain in the form of an ellipsoid of revolution showed that the elongation of a grain and forces (or stresses) applied in addition to the magnetic field can significantly change the position of the effective easy axis and the critical field of the grain magnetic moment, i.e. the magnetic state of an individual particle. The nonmonotonic variation in the critical field due to an external effect can lead, in the presence of a magnetic field, to the formation of a remanence additional to the normal remanent magnetization (NRM) or, in the absence of the field, to the NRM destruction. Various types of remanent magnetization are compared within the framework of the model of interacting heterogeneous single-domain particles. It is shown that, in diagnosing thermoremanent (TRM) and chemical magnetizations, information on the ratios of these magnetizations to the anhysteretic magnetization should be complemented by data on the coercivity and the intensity of the magnetostatic interaction of grains. The possible variation in the spontaneous magnetization with time due to diffusion processes can lead to stabilization of the vector $\mathbf{I}_{s}$ and a rise in the magnetic moment of the system (diffusive viscous magnetization). Moreover, a mechanism of the chemical magnetization formation differing from the crystallization mechanism and analogous to the TRM formation mechanism is possible. Results of this work can be helpful in solving geophysical problems and creating new magnetic minerals. INDEX TERMS: 1500

Geomagnetism and Paleomagnetism; 1518 Geomagnetism and Paleomagnetism: Magnetic fabrics and anisotropy; 1599 Geomagnetism and Paleomagnetism: General or miscellaneous; KEYWORDS: Magnetic ordering, phase transitions, diagnosis of remanence types.

Citation: Afremov, L. L., and V. I. Belokon (2005), Magnetic properties of single-domain particles and the rock remanence, Russ. J. Earth. Sci., 7, ES4004, doi:10.2205/2005ES000184.

\section{Introduction}

[2] The remanent magnetization of rocks is a metastable magnetic state of a system of ferromagnetic (antiferromagnetic) particles in a nonmagnetic matrix; this state preserves,

Copyright 2005 by the Russian Journal of Earth Sciences. ISSN: 1681-1208 (online) to an extent, the information on rock transformations due to various factors (the geomagnetic field, temperature, pressure, chemistry, and time). The reconstruction of effects applied to a rock (the solution of an inversion problem) can be effected only if the response of the system of ferromagnetic particles to each of these factors is clearly understood. In the presence of the external field, this response involves the formation of a remanent magnetization, often carrying pertinent information. Therefore, rock magnetism studies have 
been often applied to the construction of theories describing the properties of various types of remanent magnetization under conditions of a weak magnetic field (of the same order as the Earth's field) and significant effects of other factors [Khramov et al., 1982; Nagata, 1961; Neel, 1955; Petrova, 1961].

[3] The earliest studies in this direction used the simplest models of a ferromagnetic rock, each describing a single type of magnetization. However, researchers arrived at the understanding of the fact that interrelations between various types of magnetization should be examined in terms of a general model because the demagnetization technique aimed at the removal of secondary magnetization inevitably affected the primary magnetization. In particular, such was the Preisach-Neel model supplemented with concepts of interrelated hysteretic and thermal-activation processes [Belokon et al., 1973; Sholpo, 1977]. In our opinion, this model fits best a system of single-domain (SD) or pseudosingle-domain (PSD) particles such that each particle can be brought into correspondence with an elementary rectangular hysteresis cycle with critical fields of normal $(a)$ and reversed (b) magnetizations. In this case an initial isolated SD or PSD particle has a symmetric hysteresis loop with a critical field $H_{0}$ corresponding to the magnetic anisotropy: $a=H_{0}+H$ and $b=H_{0}-H$, where $H$ is the random field of interaction with other particles. Evidently, the calculation of the fields $H_{0}$ and $H$, as well as the spontaneous magnetization $I_{s}$, is critical for the construction of the Preisach-Neel model, because the response of a ferromagnetic grain to external effects is determined by the dependence of $I_{s}, H_{0}$ and $H$ on their intensity. In this work, based on the simplest models, we examine the response of small ferromagnetic grains ("elements" of the Preisach-Neel model) to external effects, taking into account lattice imperfections and chemical heterogeneity of ferromagnetic material.

\section{Interaction Between Particles, Phase Transitions, and Spontaneous Magnetization}

[4] The interaction between particles randomly scattered in the grain volume or between grains randomly distributed in a nonmagnetic matrix can be conveniently analyzed in terms of the distribution function of random fields used for the first time by Chandrasekhar [1943] (see also [Shcherbakov, 1979]). Such a function was calculated in [Belokon and Semkin, 1992, 1993] with reference to the spin exchange interaction in a lattice with impurity atoms or vacancies. The following three results obtained in these studies are significant for the description of magnetic properties of rock grains, whose lattice is strongly imperfect.

[5] (i) There exists a minimum concentration $n_{0}$ of interacting particles (a percolation limit) enabling the formation of spontaneous magnetization.

[6] (ii) The spontaneous magnetization and the Curie point depend on $n$ (at $n>n_{0}$ ) and tend toward ultimate values of a "pure" ferromagnetic material.
[7] (iii) Active diffusion of particles should apparently lead to a local increase in the concentration to limits $n>n_{0}$, because the concentration phase transition is absent if diffusion is taken into account.

[8] These considerations imply that the Curie point $T_{c}$ should change (rise) with an increase in the local concentration of (for example, during chemical reactions) and a chemical magnetization should form, as the Curie point rises from $T_{c}<T$ to $T_{c}>T$ [Belokon et al., 1995], where $T$ is the temperature during which the reaction proceeds. A more general approximate approach to the estimation of the distribution function of random interaction fields is formulated in [Belokon and Nefedev, 2001, 2002], where the following conclusions were drawn.

[9] (a) The distribution density of random interaction fields has the form of a normal distribution:

$$
W(H)=\frac{1}{B \sqrt{\pi}} \exp \frac{\left(H-\widehat{H}_{0} M\right)^{2}}{B^{2}},
$$

where

$$
\begin{gathered}
M=\int \tan \frac{\mu H}{k T} W(H) d H, \\
\widehat{H}_{0}=n \int \varphi d V, B=2 n \int \varphi^{2} d V
\end{gathered}
$$

$n$ is the number of interacting particles in a unit volume, and $\varphi(\mathbf{r})$ is the interaction law.

[10] (b) The replacement of the exact distribution function $\frac{1}{\sqrt{\pi}} \exp ^{-x^{2}}$ by a steplike function of the form

$$
W(x)=\left\{\begin{array}{l}
0,-1<x \\
\frac{1}{2},-1 \leq x \leq 1 \\
0, x>1
\end{array}\right.
$$

leads to insignificant errors in calculations of mean values.

[11] (c) The system can experience phase transitions to either the spin glass state or the ferromagnetic state, depending on the concentration $n$ and the interaction intensity. In particular, grains and their associations depleted in ferromagnetic atoms can have an ordering of the spin glass type, which is very important in studying viscous processes. If the projection $H$ of the dipole-dipole interaction field in a system of single-domain grains is taken as the function $\varphi(\mathbf{r})$, we obtain the distribution function

$$
\begin{gathered}
W(H)=\frac{1}{B \sqrt{\pi}} \exp -\frac{H^{2}}{B^{2}}, \\
M=0, B^{2}=\frac{4 \pi}{r_{0}^{3}} n m^{2},
\end{gathered}
$$

where $r_{0}$ is the characteristic sizes of grains and $m$ is the magnetic moment of a grain.

[12] However, we should note that the distribution function of the interaction field modulus $|\mathbf{H}|$ should be known in order to calculate the field $a$ and $b$ [Belokon, 1985]: 


$$
\gamma(|\mathbf{H}|)=-\left.2|\mathbf{H}| \frac{d W}{d H}\right|_{H=|\mathbf{H}|}
$$

[13] With a known function of distribution of particles over critical fields $f\left(H_{c}\right)$, the density of particles in the PreisachNeel diagram can be found:

$$
P(a, b)=\frac{1}{2} f\left(\frac{a+b}{2}\right) \gamma\left(\frac{b-a}{2}\right),
$$

enabling the calculation of any type of remanence [Belokon, 1985].

[14] Other properties of the behavior of single-domain particles related to their significant heterogeneity and the possible concurrent types of magnetic anisotropy are discussed below.

\section{Magnetic States of Single-Domain Grains}

\subsection{Chemically Homogeneous Single-Domain Grains}

[15] The magnetic state stability is largely controlled by factors unrelated to the external field and giving rise to various anisotropy effects. Along with the natural crystalline form anisotropy, the stress, diffusion, exchange, surface, and other types of anisotropy that are comparable in value with the natural anisotropy can be present in a real ferromagnetic material.

[16] Below, as an example, we address a uniaxial homogeneously magnetized grain having the shape of an ellipsoid of revolution and neglect all types of anisotropy except the natural crystalline and form anisotropies.

[17] In this case, as was shown in [Afremov and Belokon, 1976, 1977], if the dimensionless constants of crystalline and form anisotropy are positive $\left(k_{A}>0\right.$ and $k_{N}>0$, respectively), the magnetic moment of a grain is oriented along the effective axis of anisotropy, making the angle

$$
\widehat{\delta}=\frac{1}{2} \arctan \frac{k_{N} \sin 2 \alpha}{k_{A}+k_{N} \cos 2 \alpha}
$$

with the axis of the crystalline anisotropy, and its orientation in the equilibrium state can be either parallel or antiparallel to the chosen direction. The critical field $H_{0}$ of the transition of one equilibrium state into another is determined by the effective constant of anisotropy $K$ :

$$
H_{0}=K I_{s}=I_{s} \sqrt{k_{A}^{2}+k_{N}^{2}+2 k_{A} k_{N} \cos 2 \alpha} .
$$

[18] This result is remarkable because it indicates the presence of a spectrum of critical fields in a system of particles differing in $k_{N}$ and $\alpha$. Moreover, of the entire set of particles with fixed angle $\alpha$, particles meeting the condition $k_{N}=-k_{A} \cos 2 \alpha$ possess the weakest critical field $H_{0}=k_{A} I_{s} \sin 2 \alpha$.
[19] Analysis of equilibrium magnetic moment states in grains with $k_{A} \geq 0, k_{N} \leq 0$ and $k_{A} \leq 0, k_{N} \geq 0$ [Afremov and Belokon, 1979] shows that equilibrium orientations of the magnetic moment are determined (??) in these cases in the same way as at $k_{A} \geq 0, k_{N} \geq 0$ whereas, in a magnetic field directed along such an effective easy axis, an irreversible change in the magnetic moment takes place upon reaching the critical field

$$
H_{0}=I_{s}\left(k_{A}+k_{N}+\sqrt{k_{A}^{2}+k_{N}^{2}+2 k_{A} k_{N} \cos 2 \alpha}\right) .
$$

[20] With $k_{A} \leq 0, k_{N} \leq 0$, the effective easy axis coincides with the intersection of "easy" planes specified by the anisotropy of form and the natural crystalline anisotropy. The orientation of the grain magnetic moment changes irreversibly upon reaching the critical field [Afremov and Belokon, 1979]

$$
H_{0}=I_{s}\left(\left|k_{A}\right|+\left|k_{N}\right|-\sqrt{k_{A}^{2}+k_{N}^{2}+2 k_{A} k_{N}}\right) .
$$

The study of nonspherical particles under uniaxial compression [Afremov and Belokon, 1980a, 1980b] showed that the magnetic moment of a grain is oriented along an effective axis whose position and the effective constant of anisotropy are determined by anisotropy of the crystalline, form and stress types. If the external field is applied along the effective axis and makes an angle $\beta$ with the axis of the crystalline anisotropy, the position of the effective axis and the critical field of the grain magnetic moment remagnetization depend on the above types of anisotropy as follows:

$$
\begin{gathered}
\delta=\frac{1}{2} \arctan \times \\
\frac{k_{N} \sin 2 \alpha+\lambda_{4} \sigma \sin 2 \beta}{k_{A}+k_{N} \cos 2 \alpha+\left(\lambda_{1}+\lambda_{2}\right) \sigma+\left(\lambda_{1}-\lambda_{2}\right) \sigma \cos 2 \beta}, \\
H_{0}=K I_{s}=I_{s}\left\{\left[k_{A}+k_{N} \cos 2 \alpha\right.\right. \\
\left.+\left(\lambda_{1}+\lambda_{2}\right) \sigma+\left(\lambda_{1}-\lambda_{2}\right) \sigma \cos 2 \beta\right]^{2} \\
\left.+\left[k_{N} \sin 2 \alpha+\lambda_{4} \sigma \sin 2 \beta\right]^{2}\right\}^{1 / 2},
\end{gathered}
$$

where $\lambda_{i}$ are magnetostriction constants of a uniaxial crystal and $\sigma$ denotes uniaxial stresses. If the stresses are perpendicular to the plane $\left(k_{A}, k_{N}\right)$, we have

$$
\begin{gathered}
\widehat{\delta}=\frac{1}{2} \arctan \frac{k_{N} \sin 2 \alpha}{k_{A}+k_{N} \cos 2 \alpha-2 \lambda_{3} \sigma}, \\
H_{0}=K I_{s}= \\
I_{s} \sqrt{\left(k_{A}-2 \lambda_{3} \sigma\right)^{2}+k_{N}^{2}+2\left(k_{A}-2 \lambda_{3} \sigma\right) k_{N} \cos 2 \alpha} .
\end{gathered}
$$

As in the case of two types of anisotropy, the critical field can behave nonmonotonically with variations in the applied stresses. With the stresses oriented parallel to the plane $\left(k_{A}, k_{N}\right)$, the critical field reaches the minimum value $H_{0 \text { min }}=k_{A} I_{s} \sin \left(2 \alpha-\psi_{0}\right)$ at 


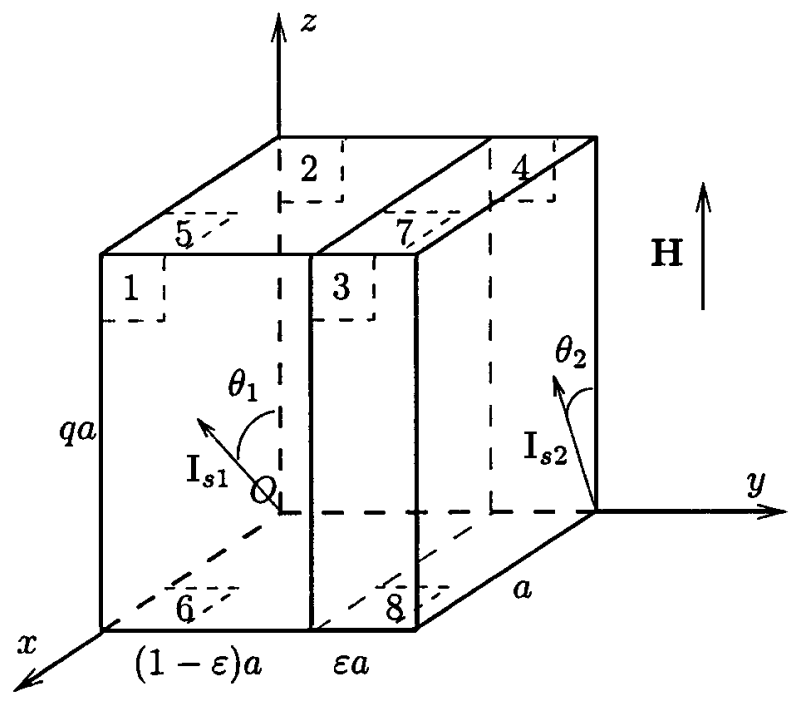

Figure 1. Illustration of the two-phase particle model with the parallel orientation of phases.

$$
\begin{gathered}
\sqrt{\left.\left[k_{A}+\left(\lambda_{1}+\lambda_{2}\right) \sigma+\left(\lambda_{1}-\lambda_{2}\right) \sigma \cos 2 \beta\right]^{2}+\lambda_{4} \sigma \sin 2 \beta\right]^{2}} \\
=-k_{N} \cos \left(2 \alpha-\psi_{0}\right)
\end{gathered}
$$

where

$$
\psi_{0}=\arctan \frac{\lambda_{4} \sigma \sin 2 \beta}{k_{A}+\left(\lambda_{1}+\lambda_{2}\right) \sigma+\left(\lambda_{1}-\lambda_{2}\right) \sigma \cos 2 \beta} .
$$

If the stresses are perpendicular to the plane $\left(k_{A}, k_{N}\right)$, the critical field attains the minimum value $H_{0}=k_{N} I_{s} \sin 2 \alpha$ at $k_{A}-2 \lambda_{3} \sigma=-k_{N} \cos 2 \alpha$.

[21] Summarizing, we can note the following.

[22] (1) The grain elongation and uniaxial stresses can change significantly the magnetic state of a particle.

[23] (2) The orientation of the effective easy axis and the critical field of an irreversible change in the magnetic moment of a grain are basically dependent on the mutual orientations of the uniaxial stresses and the axes of the crystalline and form anisotropies, as well as on the signs of $\sigma, k_{A}$ and $k_{N}$.

[24] (3) The application of any external force in the absence of a magnetic field can lead to the destruction of remanent magnetization.

\subsection{Magnetic States of Two-Phase Particles}

[25] It is known that chemical processes (such as oxidation or decomposition of a solid solution) in a magnetically ordered grain can lead to the formation of phases of different compositions and, as a result, an inhomogeneous distribution of magnetic moments. The simplest model of coexisting phases of different compositions is a particle consisting of two crystallographically uniaxial, homogeneously magnetized ferromagnetic phases and represented by a parallelepiped of a base $a^{2}$ and a height $q a$ (Figure 1). Ferromagnetic regions (phases) are characterized by the following parameters: $I_{s 1}$ and $I_{s 2}$, spontaneous magnetizations; $k_{1}$ and $k_{2}$, dimensionless constants of crystalline anisotropy; and $1-\varepsilon$ and $\varepsilon$, relative volumes of the first and second phases, respectively. For simplicity, we assume that the vectors $I_{s 1}$ and $I_{s 2}$ lie in the plane $x 0 z$ and the crystalline anisotropy axes of both ferromagnetic phases are parallel to the $0 z$ axis. The particle is in an external magnetic field $H$ directed along the $0 z$ axis.

[26] In our analysis, we neglect the magnetoelastic interphase interaction, which can be more or less valid only in the case of a strongly disordered distribution of magnetic atoms in the boundary layer.

[27] 3.2.1. Equilibrium grain states in an external magnetic field. Analysis of the free energy of a grain [Afremov and Belokon, 1996a, 1996b] shows that, in the accepted approximation (in the absence of an external magnetic field), a two-phase particle can exist in one of the following states:

[28] first $(\uparrow \uparrow)$ state: the magnetic moments of both phases are parallel and directed along the $0 z$ axis;

[29] second $(\uparrow \downarrow)$ state: the magnetizations of the phases are antiparallel, with the magnetic moment of the first phase $m_{1}$ being directed along the $0 z$ axis;

[30] third $(\downarrow \downarrow)$ state: the magnetic moments of the phases are parallel and opposite in direction to the $0 z$ axis; and

[31] fourth $(\downarrow \uparrow)$ state: the magnetic moments of the phases are antiparallel, with the first phase being magnetized in the direction opposite to the $0 z$ axis.

[32] If the magnetostatic interaction between the phases prevails over the exchange interaction, $N_{21}^{*}=N_{21}-A^{*}>0$ ( $N_{i k}$ are the demagnetizing coefficients, $A^{*}=2 A_{i n} / \delta a I_{s 1} I_{s 2}$, $A_{\text {in }}$ is the exchange constant, and $\delta$ is the width of the interphase transition zone having the same order of magnitude as the lattice parameter), then the first and third states are metastable because the free energy of the grain $F=N_{21} I_{s 1} I_{s 2}$ in these states is higher than the free energy in the second and fourth states $F=-N_{21} I_{s 1} I_{s 2}$. The second and fourth states are metastable if $N_{21}^{*}<0$.

[33] 3.2.2. Equilibrium states of a grain in an external magnetic field. The transitions, for example, from the third to the second or the fourth states are associated with a rotation of the magnetic moment of one of the phases, and the transition fro the third to the first states is associated with a rotation of the total moment of a grain.

[34] Analysis of the grain free energy shows that the thirdto-second state transition occurs in the field [Afremov and Panov, 1996a, 1996b]

$$
H_{c 1}^{(\uparrow \uparrow)}=k_{1} I_{s 1}+\frac{2 N_{11} I_{s 1}-N_{21}^{*} I_{s 2}}{1-\varepsilon} .
$$

Similar calculations yield the following critical fields:

$$
H_{c 2}^{(\uparrow \uparrow)}=k_{2} I_{s 2}+\frac{2 N_{22} I_{s 2}-N_{21}^{*} I_{s 1}}{\varepsilon}
$$

for the third-to-fourth state transition, 


$$
H_{c 1}^{(\uparrow \downarrow)}=k_{1} I_{s 1}+\frac{2 N_{11} I_{s 1}+N_{21}^{*} I_{s 2}}{1-\varepsilon}
$$

for the fourth-to-first state transition,

$$
H_{c 2}^{(\uparrow \downarrow)}=k_{2} I_{s 2}+\frac{2 N_{22} I_{s 2}+N_{21}^{*} I_{s 1}}{\varepsilon}
$$

for the second-to-first state transition,

$$
H_{c 3}^{(\uparrow \uparrow)}=\frac{a_{1} I_{s 1}^{2}+a_{2} I_{s 2}^{2}+2\left(N_{12}-N_{21}\right) I_{s 1} I_{s 2}}{(1-\varepsilon) I_{s 1}+\varepsilon I_{s 2}}
$$

for the third-to-first state transition, and

$$
H_{c 3}^{(\uparrow \downarrow)}=\frac{a_{1} I_{s 1}^{2}+a_{2} I_{s 2}^{2}-2\left(N_{12}-N_{21}\right) I_{s 1} I_{s 2}}{\left|(1-\varepsilon) I_{s 1}-\varepsilon I_{s 2}\right|}
$$

for the second-to-fourth state transition.

[36] 3.2.3. The temperature effect on magnetic states of heterogeneous particles. [36] The above approach is applicable only to the study of magnetic states at $T=0$. If $T \neq 0$, the role of thermal fluctuations increases with decreasing size of particles, and an ensemble of ferromagnetic particles becomes superparamagnetic: its behavior is similar to that of a paramagnetic gas. A distinction is that, depending on the height of the potential barrier between equilibrium states, the magnetic moment of a particle can exist for a certain time $\tau$ in one of the states.

[37] Because of the small volume of particles, one may expect that, due to thermal fluctuations of magnetic moments of phases, transitions from one equilibrium state into another can be realized in a field $\mathbf{H}$ that is smaller than any of the critical fields (15)-(20).

[38] The reorientation of the magnetic moment of a phase is determined by the height of the potential barrier $E_{i k}$ separating the $i$ th and $k$ th states. The frequency of the $i-k$ state transition is expressed as

$$
W_{i k}=f_{0} \exp \left(-\frac{E_{i k}}{k_{B} T}\right) .
$$

Here $f_{0} \approx 10^{7}-10^{10} \mathrm{~s}^{-1}$ is the characteristic frequency of "attempts" to overcome the potential barrier, $k_{B}$ is the Boltzmann constant, and $E_{i k}=F_{i k \max }-F_{i k \text { min }}$ where $F_{i k \text { min }}$ is the free energy of the $i$ th equilibrium state characterizing the particle before the transition and $F_{i k \text { max }}$ is the maximum free energy separating the $i$ th and $k$ th states.

[39] If the transition probability from one state to another is known, four equations of "motion" can be constructed for the vector of states:

$$
\frac{d N_{i}}{d t}=\sum_{k \neq i}^{4}\left(-W_{i k} N_{k}+W_{k i} N_{k}\right)
$$

Considering the normalization condition $N_{1}+N_{2}+N_{3}+N_{4}=$ 1 , these equations can be reduced to the form

$$
\frac{d \tilde{N}}{d t}=\widetilde{W} \widetilde{N}+V
$$

where

$$
\begin{aligned}
& \widetilde{W}_{i k}=\left\{\begin{array}{cc}
-\sum_{j \neq i}^{4} W_{i j}-W_{4 i}, i=k, \\
W_{i k}-W_{4 i}, & i \neq k
\end{array}\right. \\
& \widetilde{N}(t)=\left(\begin{array}{c}
N_{1}(t) \\
N_{2}(t) \\
N_{3}(t)
\end{array}\right), V=\left(\begin{array}{c}
W_{41} \\
W_{42} \\
W_{43}
\end{array}\right), N_{0}=\left(\begin{array}{c}
N_{01} \\
N_{02} \\
N_{03}
\end{array}\right) .
\end{aligned}
$$

The solution of (23) can be conveniently represented with the use of a matrix exponential:

$$
\widetilde{N}(t)=N_{0} \exp (\widetilde{W} t)+N \int_{0}^{t} \exp \{\widetilde{W}(t-\tau)\} d \tau
$$

With the known vector of the initial state

$$
n_{0}=\left\{N_{01}, N_{02}, N_{03}, N_{04}\right\}
$$

relations (15)-(25) completely determine not only the magnetic state population but also the magnetization of the ensemble of two-phase particles [Afremov and Panov, 1998a, 1998b, 2004].

\section{Relation between Various Types of Remanent Magnetization}

[40] The dependence of magnetic states of single-domain grains on various factors (temperature, pressure, chemical composition, time, magnetostatic interaction of particles, and others) inferred above can be used to describe and compare various types of the remanent magnetization in terms of a unified model.

[41] Thus, comparison of thermoremanent $\left(I_{r t}\right)$ and anhysteretic $\left(I_{r i}\right)$ magnetizations in ensembles of single-domain particles coinciding (narrow spectrum) and differing (wide spectrum) in coercivity [Afremov and Kharitonsky, 1986a, 1986b] shows that $R_{t}=I_{r t} / I_{r i}<1$ for a system of weakly interacting grains and $R_{t}>2$ for strongly interacting grains (Figure 2). The dependence of the ratio between chemical and anhysteretic magnetization $R_{c}=I_{r c} / I_{r i}$ on the factor $F$ defined by Sholpo [1977] is plotted in Figure 3. The curves $R_{c}=R_{c}(F)$ are similar in behavior to $R_{t}=R_{t}(B)$, which is quite natural because $F \approx B / H_{\max }$ at $F \ll 1$.

[42] Comparing $R_{t}$ and $R_{c}$, it is easy to show that, with increasing $H_{c \max }$, the parameter $R_{c}$ reaches an ultimate value at greater values of $B$ than the parameter $R_{t}$ does. This is due to the fact that the chemical and thermoremanent magnetizations are mainly due to, respectively, low- and highcoercivity grains. Note also that, in the experimentally observed range of interaction fields $(B \approx 3-30$ Oe) [Ivanov et al., 1981; Ivanov and Sholpo, 1982], the maximum value of $R_{c}$ is $R_{c} \approx 1$, whereas $R_{t}$ depends on coercivity. 


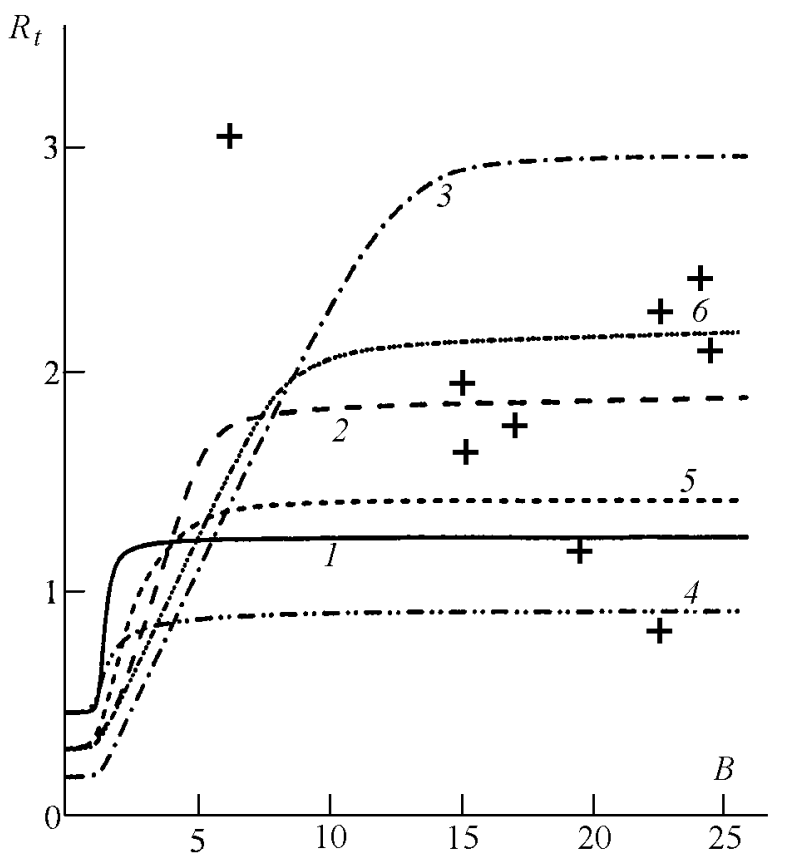

Figure 2. Dependence of $R_{t}$ on the interaction field $B$. Curves 1, 2, and 3 relate to a narrow spectrum with $H_{0}$ $=100,400$, and 1600 Oe, respectively. Curves 4, 5, and 6 relate to a wide spectrum with $H_{\max }=100,400$, and 1600 Oe, respectively. The crosses are experimental points [Ivanov et al., 1981].

[43] Knowing the response of each particle to an external effect (variations in $H_{c}$ and $I_{s}$ and the corresponding displacements of representative points in a diagram), we can easily calculate all of the aforementioned types of remanent magnetization and establish the relations between them.

[44] The function $\gamma(|\mathbf{H}|)$ can also be applied for the estimation of detrital magnetization in a system of large particles whose orientation is modified by a magnetic interaction field rather than temperature. This estimate is directly related to the so-called cluster model of depositional magnetization developed in [Shashkanov et al., 1989, 2003].

[45] Using the distribution density given by (4), one can show [Belokon and Nefedev, 2001] that, before the decrease in the tilt angle of a certain portion $P$ of elongated or flattened particles, the detrital magnetization is given by the formula

$$
\mathbf{I}_{r 0} \approx n m \frac{4}{3} \pi \frac{\mathbf{H}}{B}
$$

where $\mathbf{H}$ is the external field. After the particles become less tilted, we have

$$
\widetilde{I}_{r_{0}, x}=\frac{4}{3}(1-p) \frac{H}{B} n m \sin \vartheta_{0}+\frac{4}{\pi} \frac{2 \vartheta_{0}}{\pi} p \frac{4}{3} \pi n m \frac{H}{B}
$$

$$
\begin{aligned}
\widetilde{I}_{r 0, z} & =\frac{4}{3}(1-p) n m \cos \vartheta_{0}, \\
\tan \widetilde{\vartheta}_{0} & =\tan \vartheta_{0}+\frac{8 \vartheta_{0}}{\pi^{2}} \frac{p}{1-p} .
\end{aligned}
$$

Here $\left(\frac{\pi}{2}-\vartheta_{0}\right)$ is the inclination of $\tilde{I}_{r 0}$ and $\tilde{\vartheta}_{0}-\vartheta_{0}$ is its

[46] In conclusion, we can note the following.

[47] (1) Knowledge of values of $R_{t}$ and $R_{c}$ is insufficient for the identification of thermoremanent and chemical magnetizations in an ensemble of single-domain particles. This identification requires additional information on the coercivity and the intensity of magnetostatic grain interaction that can be obtained from laboratory experiments.

[48] (2) Spontaneous magnetization can change with time due to diffusion processes [Afremov and Belokon, 1972]. This can lead to stabilization of the vector $\mathbf{I}_{s}$ and a rise in the magnetic moment of the system (diffusion-induced viscous magnetization).

[49] (3) The formation of chemical magnetization can be interpreted in terms of a mechanism [Belokon et al., 1995] that is an analogue of the thermoremanent magnetization formation mechanism and differs from the crystallization mechanism proposed by Haig [1962].

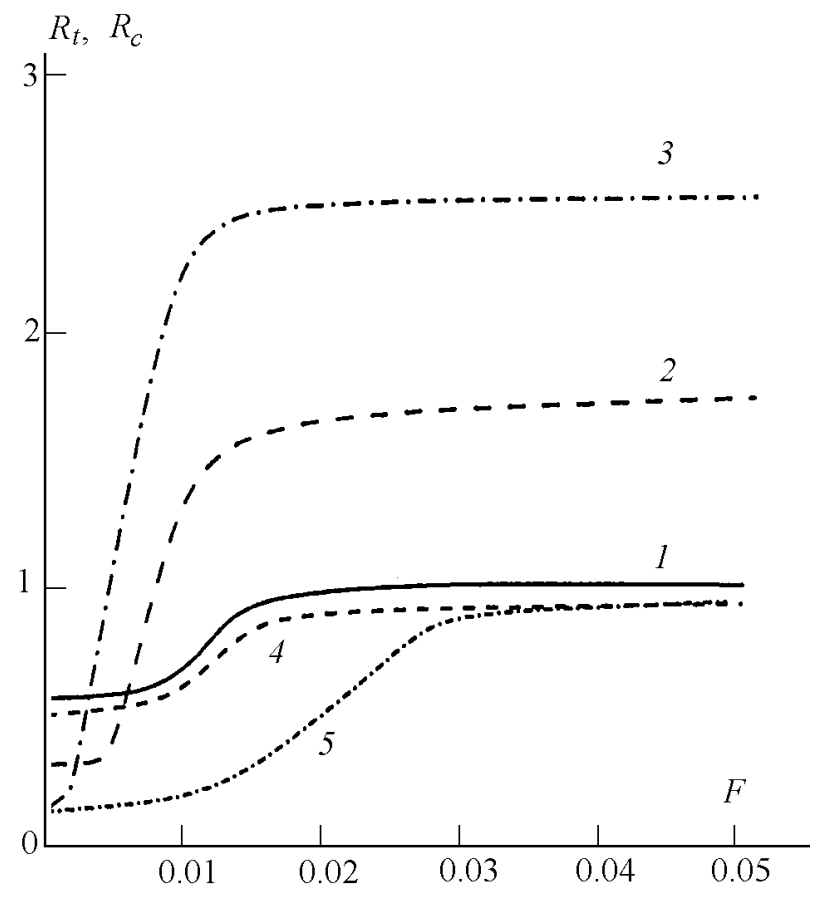

Figure 3. $R_{t}$ versus $F$ (curves 1,2 , and 3 with the respective values $H_{\max }=100,400$, and $1600 \mathrm{Oe}$ ) and $R_{c}$ versus $F$ (curves 4 and 5 with the respective values $H_{\max }=100$ and 400 Oe). 


\section{Conclusion}

[50] The model of interacting single-domain particles underlying the analysis of relations between various types of magnetization is, in a certain aspect, similar to the model of an ideal gas in which every atom is represented, in a first approximation, by a particle that is only capable of energy and momentum exchange with other particles. It is remarkable that, even in terms of this simple model, one can obtain the Mendeleev-Clapeyron equation, having a rather wide application area. A simple technique incorporating a finite volume of atoms and their possible interaction yields the Van der Waals equation predicting vapor-liquid transitions. Of course, a more realistic description of an atom as a complex quantum-mechanical system appears to be preferable, but numerical problems involved in this approach are evident; it is also evident that some reasonable restrictions are inevitable in incorporating properties of the atom, which is a main constituent of the kinetic theory of gases. A similar situation is observed in the case of the system of interacting single-domain particles whose properties are fairly complex, as is evident from the results of this paper. Therefore, it is natural to accept, as a first approximation, the simplest model of a single-domain particle and introduce additional restraints only if model results diverge significantly with experimental data. In our opinion, the results that we obtained from this detailed study of properties of singledomain particles can be useful for gaining insights into such well-known phenomena as the stabilization of the magnetic moment with time, the metastability of magnetic states in rocks, the pressure-induced destruction of remanent magnetization, and so on; these phenomena cannot be interpreted in terms of the simplest assumptions on the properties of $I_{r}, H_{0}$ and the interaction field $H$.

\section{References}

Afremov, L. L., and V. I. Belokon (1972), Evidence for the diffusion aftereffect in rocks, Izv. Phys. Solid Earth, Fiz. Zemli (in Russian), 8(11), 91.

Afremov, L. L., and V. I. Belokon (1976), Remanent magnetization in an ensemble of single-domain grains near a zero anisotropy constant, Izv. Phys. Solid Earth, Fiz. Zemli (in Russian), 12(11), 111.

Afremov, L. L., and V. I. Belokon (1977), On the calculation of the critical field of single-domain grains, Izv. Phys. Solid Earth, Fiz. Zemli (in Russian), 13(3), 122.

Afremov, L. L., and V. I. Belokon (1979), On the calculation of the remanent magnetization in a system of single-domain particles, Izv. Phys. Solid Earth, Fiz. Zemli, 15(4), 122.

Afremov, L. L., and V. I. Belokon (1980a), The effect of mechanical stresses on magnetic states and the critical field of single-domain particles, Izv. Phys. Solid Earth, Fiz. Zemli (in Russian), 16(2), 101.

Afremov, L. L., and V. I. Belokon (1980b), The effect of mechanical stresses on the processes of remanence acquisition and destruction in a system of single-domain particles, Izv. Phys. Solid Earth, Fiz. Zemli (in Russian), 16(8), 101.

Afremov, L. L., and P. V. Kharitonsky (1986a), Calculation of the crystallization magnetization for a system of interacting single-domain particles, in Paleointensity: Physical Principles and Methods of Study (in Russian), p. 3, DVU, Vladivostok.
Afremov, L. L., and P. V. Kharitonsky (1986b), Some properties of the crystallization magnetization in a system of interacting single-domain particles, in Paleointensity: Physical Principles and Methods of Study (in Russian), p. 11, DVU, Vladivostok.

Afremov, L. L., and A. V. Panov (1996a), Theory of magnetization of two-phase superparamagnetic particles I, Magnetic States, FMM (in Russian), 82(5), 5.

Afremov, L. L., and A. V. Panov (1996b), Theory of magnetization of two-phase superparamagnetic particles II, Modeling of magnetization processes, FMM (in Russian), 82(5), 17.

Afremov, L. L., and A. V. Panov (1998a), Modeling the magnetization of an Ensemble of chemically inhomogeneous superparamagnetic titanomagnetite particles, Izv. Phys. Solid Earth (in Russian), 34, 164.

Afremov, L. L., and A. V. Panov (1998b), Magnetic states and hysteresis characteristics of fine magnetite particles, FMM (in Russian), 86(3), 65.

Afremov, L. L., and A. V. Panov (2004), Remanent Magnetization of Ultradisperse Magnetic Substances (in Russian), 192 pp., DVU, Vladivostok.

Belokon, V. I. (1985), On the relation between some types of remanent magnetization in an ensemble of interacting singledomain particles, Izv. Phys. Solid Earth, Fiz. Zemli (in Russian), 21(2), 123.

Belokon, V. I, V. V. Kochegura, and L. E. Sholpo (1973), Methods of Paleomagnetic Studies of Rocks (in Russian), 247 pp., Nedra, Leningrad.

Belokon, V. I., and S. V. Semkin (1992), Method of a random field in the Ising model of a diluted ferromagnet, ZhETF (in Russian), 101(10), 1254.

Belokon, V. I., and S. V. Semkin (1993), Method of a random field in the ferromagnetism theory of binary alloys, ZhETF (in Russian), 104(5), 3784.

Belokon, V. I., A. S. Kuchma, and I. V. Soppa (1995), Remanence acquisition during chemical transformations accompanied by a change in the Curie point of the reaction products, Izv. Phys. Solid Earth, Fiz. Zemli (in Russian), 31(11), 84

Belokon, V. I., and K. V. Nefedev (2001), The distribution function of random interaction fields with concurrent exchange interaction, ZhETF (in Russian), 120(1(7)), 156.

Belokon, V. I., and K. V. Nefedev (2002), Magnetic phase transitions in amorphous systems with concurrent exchange interaction, Fiz. Tverd. Tela (in Russian), 44(9), 1632.

Belokon, V. I., and K. V. Nefedev (2004), Inclination error and cluster theory of detrital magnetization, in Proceedings of the International Seminar "Paleomagnetism and Magnetism of Rocks" (in Russian), p. 69, Kazanskii Universitet, Kazan.

Chandrasekhar, S. (1943), Stochastic problems in physics and astronomy, Rev. Mod. Phys., 15, 1.

Haig, G. (1962), Acquisition of remanence due to chemical alterations, in Paleomagnetism, edited by G. N. Petrova (in Russian), p. 67, IL, Moscow.

Ivanov, V. A., I. A. Khaburzaniya, and L. E. Sholpo (1981), The Preisach diagram as a tool for the identification of singleand multidomain grains in rock samples, Izv. Phys. Solid Earth, Fiz. Zemli (in Russian), 17(1), 55.

Ivanov, V. A., and L. E. Sholpo (1982), Numerical criteria of single- and multidomain states in rock minerals, Izv. Phys. Solid Earth, Fiz. Zemli (in Russian), 18(8), 84.

Khramov, A. N. (1982), Paleomagnetology (in Russian), 312 pp., Nedra, Leningrad.

Nagata, T. (1961), Rock-Magnetism, 190 pp., Maruzen, Tokyo. Neel, L. (1955), Some theoretical aspects of rock-magnetism, Adv. Phys., 4(14), 191.

Petrova, G. N. (1961), Laboratory Estimation of the Remanence Stability in Rocks (in Russian), 96 pp., AN SSSR, Moscow.

Shashkanov, V. A., A. I. Novoselov, and A. V. Smirnov (1989), Mechanisms of detrital magnetization acquisition by magnetitebearing sedimentary rocks, Izv. Phys. Solid Earth, Fiz. Zemli (in Russian), 25(8), 45. 
Shashkanov, V. A., E. V. Isupova, and P. V. Dubrovin (2003), Elimination of the inclination error in the detrital magnetization of sedimentary rocks, Izv. Phys. Solid Earth, 39, 749.

Shcherbakov, V. P. (1979), On the susceptibility of interacting superparamagnetic grains, Fiz. Tverd. Tela (in Russian), $48(6), 1134$
Sholpo, L. E. (1977),

Application of Rock Magnetism to Geological Problems (in Russian), 97 pp., Nedra, Leningrad.

L. L. Afremov and V. I. Belokon, Far East State University, 8 Sykhanova ul., Vladivostok, 690950 Russia 